\title{
Tiempo en rango terapéutico (TRT) en clínica de anticoagulación Reportes de eventos adversos y factores asociados a bajo TRT
}

\section{Time in therapeutic range (TTR) in anticoagulation clinic Reports of adverse events and factors associated with low TTR}

\author{
Harold Miranda, Santiago Osorio, Diana Patricia Giraldo, \\ Julieta Duque, John Ubeimar Cataño, luis Ignacio Tobón, \\ Fernando Antonio Mejía, Felipe Gómez, Mario Bedoya, \\ Diana Carolina Inguilan • Medellín (Colombia)
}

\section{Resumen}

En el tratamiento de anticoagulación con warfarina, la reducción de eventos tromboembólicos debe ser valorada con el riesgo de sangrado. El tiempo de rango terapéutico (TRT) por el método de Rosendaal es una herramienta que valora la calidad en la monitorización de la terapia anticoagulante y se correlaciona con presencia de eventos tromboembólicos o sangrados. En este estudio se describe el tiempo de rango terapéutico $\left(\mathrm{TRT}_{\mathrm{R}}\right)$, los factores relacionados con menor $\left(\mathrm{TRT}_{\mathrm{R}}\right)$ y los efectos adversos presentados en la clínica de anticoagulación.

Métodos y resultados: estudio descriptivo de corte transversal entre el $1^{\circ}$ de enero de 2011 y el 29 de febrero de 2012.

Fueron evaluados 2232 resultados de INR de 319 pacientes. 98550 días de seguimiento. 44\% (108) hombres, 66\% (211) mujeres, la edad promedio 60.3 años, siete visitas promedio/año, dosis semanal de warfarina $29.8 \mathrm{mg}$. La dosis semanal presenta una relación inversa con la edad, en menores de 45 años $37.9 \mathrm{mg}$ y en mayores de 75 años $22.1 \mathrm{mg}$. El TRT ${ }_{\mathrm{R}}$ incrementó de 48 - 54\%, respectivamente.

Las indicaciones para anticoagulación: fibrilación auricular (FA) 38\%(121), enfermedad tromboembólica venosa (ETEV) 35\%(112), prótesis valvulares (PV) 17.5\%(56) y embolia o trombosis arterial (EA) 9.5\%(30). 228 pacientes (71\%) presentaron un $\mathrm{TRT}_{\mathrm{R}}$ promedio del $64 \%$. (40-100) INR mayor de 5 en $2.24 \%$ e INR menor de 1.5 en $10.9 \%$.

Sangrados menores: 16 pacientes $(5 \%)$, sangrado mayor se presentó en dos pacientes $(0.65 \%)$ y un evento adverso por embolia $(0.32 \%)$.

Los factores asociados a un $\mathrm{TRT}_{\mathrm{R}}$ bajo fueron: sexo masculino, enfermedad tromboembólica venosa, uso de warfarina genérica, edad menor de 55 años, tiempo menor de un año y menos de cinco visitas.

Conclusiones: el tiempo de rango terapéutico TRT es una medición útil para establecer la eficacia de la terapia anticoagulante con warfarina. La meta de $60 \%$ en tiempo de rango terapéutico garantiza menos efectos adversos por sangrado o trombosis. Un número bajo de visitas y anticoagulación menor de un año están asociados a bajo TRT. (Acta Med Colomb 2016; 41: 42-48).

Palabras clave: clínica de anticoagulación, método de Rosendaal, warfarina, tiempo en rango terapéutico.

\footnotetext{
Abstract

In the treatment of warfarin anticoagulation, reduction of thromboembolic events must be evaluated with the risk of bleeding. Time in therapeutic range (TTR) by the method of Rosendaal is a tool that values quality monitoring anticoagulant therapy and correlates with the presence of thromboembolic events or bleeding. In this study time therapeutic range (TTR), factors associated with lower (TTR) and adverse effects presented in the anticoagulation clinic are presented.

Methods and Results: A descriptive cross-sectional study from $1^{\circ}$ January 2011 and 29th February 2012.
}

Dr. Harold Miranda Rosero: Internista, Medicina Vascular Universidad de Antioquia; Dr. Santiago Osorio: Médico General, Universidad de Antioquia; Dra. Diana Patricia Giraldo Méndez: Internista. Medicina Vascular. Hospital Universitario San Vicente Fundación; Dra. Julieta Duque Botero: Internista. Medicina Vascular Universidad de Antioquia; Dr. John Ubeimar Cataño: Internista. Medicina Vascular. Hospital Universitario San Vicente Fundación; Dr. Luis Ignacio Tobón: Internista. Medicina Vascular Universidad de Antioquia; Dr. Fernando Antonio Mejía: Internista. Medicina Vascular Universidad de Antioquia; Dr. Felipe Gómez Isaza: Internista. Medicina Vascular Universidad de Antioquia; Dr. Mario Bedoya Menco: Internista. Medicina Vascular Universidad de Antioquia; Dra. Diana Carolina Inguilan Aguirre: Estudiante Facultad de Medicina Universidad de Antioquia. Medellín (Colombia). Grupo Clínica de Anticoagulación - Hospital Universitario San Vicente Fundación. Medellín (Colombia)

Correspondencia. Dr. Harold Miranda, Medellín (Colombia).

E-mail: hamirandaro@gmail.com Recibido: 9/IX/2014 Aceptado: 29/IX/2015 
2232 results of INR of 319 patients were assessed. 98550 days follow up. 44\% (108) were men, $66 \%$ (211) women, average age 60.3 years, seven average visits / year, warfarin weekly dose of 29.8 $\mathrm{mg}$. The weekly dose has an inverse relationship with age; in patients under 45 years $37.9 \mathrm{mg}$., and in patients over 75 years, $22.1 \mathrm{mg}$. The TTR increased from 48 to $54 \%$, respectively.

Indications for anticoagulation: atrial fibrillation (AF) 38\% (121), venous thromboembolic disease (VTE) 35\% (112), prosthetic valves (PV) 17.5\% (56) and emboli or arterial thrombosis (EA) $9.5 \%$ (30). 228 patients (71\%) had a TTR average of $64 \%$. (40-100), INR greater than 5 in $2.24 \%$ and INR less than 1.5 in $10.9 \%$.

Minor bleeding: 16 patients $(5 \%)$, major bleeding occurred in two patients $(0.65 \%)$ and one adverse event of embolism $(0.32 \%)$.

The factors associated with low TTR were male gender, venous thromboembolic disease, use of generic warfarin, age less than 55 years, time shorter than one year and less than five visits.

Conclusions: TTR is a useful measurement to establish the efficacy of anticoagulant therapy with warfarin. The goal of a $60 \%$ TTR ensures fewer adverse effects from bleeding or thrombosis. A low number of visits and anticoagulation less than a year are associated with low TTR. (Acta Med Colomb 2016; 41: 42-48).

Keywords: anticoagulation clinic, Rosendaal method, warfarin, time therapeutic range.

\section{Introducción}

En los últimos cincuenta años, los antagonistas de la vitamina $\mathrm{K}$, han sido la terapia de anticoagulación disponible para la prevención primaria y secundaria de eventos tromboembólicos venosos y arteriales (1).

La warfarina ha mostrado en forma consistente ser altamente efectiva y hoy en día es utilizada por millones de personas en todo el mundo en pacientes con fibrilación auricular, enfermedad tromboembólica venosa, prótesis mecánica valvular (2-4) y otras condiciones (2-4).

Dada la complejidad de la farmacocinética y farmacodinamia, la anticoagulación con warfarina, tiene una amplia variabilidad individual en su efecto terapéutico que está dada por factores genéticos, interacciones con medicamentos, factores medioambientales, ingesta de vitamina $\mathrm{K}$, estados hipermetabólicos, enfermedades, comorbilidades y la edad que puede ser el factor más importante predictor de requerimiento de dosis (5-14).

Las interacciones con otros medicamentos, el efecto anticoagulante y la reducción de eventos tromboembólicos deben ser cuidadosamente valorados con el riesgo de sangrado asociado con esta terapia $(1,15)$.

La monitorización y el estricto control de la terapia anticoagulante reduce el riesgo de eventos tromboembólicos o sangrados. El INR (international normalized ratio) es hoy en día, el examen estandarizado para expresar los resultados del tiempo de protrombina y ha servido para validar la eficacia de la anticoagulación oral en los diferentes estudios $(16,19)$.

El resultado de INR en un momento dado, determina la efectividad del efecto anticoagulante de la warfarina y su valor se relaciona con el riesgo de sangrado o trombosis.

También podemos evaluar la calidad de la monitorización de la anticoagulación utilizando el tiempo de rango terapéutico (TRT) (20-22). El TRT por el método de Rosendaal asume una progresión linear entre dos valores de INR y calcula el INR específico para cada día (23). El TRT por el método de Rosendaal ( $\mathrm{TRT}_{\mathrm{R}}$ ) es una herramienta valiosa en la monitorización de la terapia anticoagulante con warfarina y es el método utilizado en estudios clínicos como RELY, ARISTOTLE y EINSTEIN EP, en los cuales se compara la efectividad de la anticoagulación con warfarina con los nuevos anticoagulantes (24-26).

En Colombia no hay reportes de tiempo de rango terapéutico en un servicio de anticoagulación. En el estudio RELY, pacientes con fibrilación auricular, los investigadores en Colombia reportaron $\mathrm{TRT}_{\mathrm{R}}$ del $53 \%$ (27).

En este estudio describimos las características demográficas de los pacientes en la clínica de anticoagulación, el tiempo de rango terapéutico $\left(\mathrm{TRT}_{\mathrm{R}}\right.$ ) para cada indicación de anticoagulantes orales y describimos los factores relacionados con menor tiempo en rango terapéutico $\left(\mathrm{TRT}_{\mathrm{R}}\right.$ ) y los efectos adversos presentados.

\section{Material y métodos}

Se realizó un estudio descriptivo de corte transversal sobre una población vinculada a la clínica de anticoagulación en el Hospital Universitario San Vicente Fundación de Medellín. Se incluyeron todos los pacientes que asistieron a la clínica de anticoagulación entre el $1^{\circ}$. de enero de 2011 y el 29 febrero de 2012, que tuviesen al menos cuatro visitas con reporte de INR. La clínica de anticoagulación cuenta con un registro individual de donde tomamos los datos de filiación, indicación para la terapia anticoagulante, medicamento anticoagulante original o genérico, episodios de sangrado menor o mayor, complicaciones por recurrencias o embolia y los valores de INR en cada visita con su dosis habitual día a día y el promedio de dosis semanal. Se procedió a elaborar un instrumento de recolección de la información en Excel de los datos consignados en cada tarjeta. En la 
base de datos se incluyeron las fórmulas matemáticas para realizar el cálculo de la dosis promedio semanal y se calculó el tiempo en rango terapéutico por método de interpolación de Rosendaal asumiendo un valor de INR entre 2 y 3 como rango terapéutico para todas las indicaciones.

Se realizó una prueba piloto inicial con 25 tarjetas tomadas al azar, de las cuales se extrajeron los datos registrados en las tarjetas de cada paciente y con estos datos se estandarizó el registro de los mismos, así se pudo establecer la viabilidad de este estudio y se validó el instrumento de recolección de información para el seguimiento de los pacientes en la clínica de anticoagulación.

Para el control de sesgos de información después de realizar la prueba piloto se evaluó el instrumento con el fin de evitar redundancias en la información y garantizar que se recopilasen todos los datos. El uso de formularios prediseñados para extraer los datos de las tarjetas, así como la recolección de los datos por los investigadores redujo el sesgo de información.

Se hicieron las siguientes definiciones: complicaciones por hemorragia en terapia anticoagulante y eventos trombóticos o tromboembólicos.

Complicaciones por hemorragia en terapia anticoagulante. El sangrado mayor es definido por principios generales y criterios clínicos objetivos según ISTH (International Society on Thrombosis and Haemostasis) (28), cuando produce la muerte, atenta contra la vida, causa secuelas clínicas o consume grandes e importantes recursos de la salud. Teniendo en la cuenta estos aspectos, se ha definido como sangrado mayor:

- Sangrado fatal

- Sangrado en un órgano o área crítica como: sistema nervioso central, intraocular, intraperitoneal, intraarticular, pericárdico o intramuscular que produce síndrome compartimental.
- Sangrado que causa caída de más de $2 \mathrm{~g}$ de hemoglobina, o requiere dos o más unidades de concentrado globular.

El sangrado menor clínicamente significativo o relevante es definido como aquel que no cumple criterios para sangrado mayor y que ocurre: en el tracto gastrointestinal, (excepto sangrado por hemorroides), genitourinario (hematuria macroscópica de al menos 24 horas), epistaxis que requiere intervención sea o no recurrente por lo menos de cinco minutos de duración, hematoma extenso de por lo menos $5 \mathrm{~cm}$ de diámetro, metrorragia o menorragia $(29,30)$. Los otros episodios de sangrado se consideraron menores.

Los eventos trombóticos o eventos embólicos fueron los presentados por recurrencia de enfermedad tromboembólica, embolia arterial, cardioembolia o trombos en válvula mecánica documentados por historia clínica.

Se realizó un análisis univariado en la descripción de las características de los pacientes en anticoagulación según la naturaleza de las variables. En variables cualitativas se utilizaron frecuencias absolutas y relativas (porcentaje).

En este estudio no se realiza ningún tipo de intervención farmacológica o psicológica, se cataloga como un estudio sin riesgo por la Resolución 8430 de 1993, no se requiere solicitar consentimiento informado por escrito a cada paciente. Esta investigación cumple con las normas éticas estipuladas en la Resolución de Helsinki.

\section{Resultados}

Trescientos diez y nueve pacientes cumplieron los criterios de inclusión y asistieron a la clínica de anticoagulación del $1^{\circ}$. de enero de 2011 a 29 febrero de 2012. Las características generales se observan en Tabla 1 .

Se evaluaron 2232 visitas con resultado de INR. 98550 días de seguimiento. Siete visitas promedio/año. Doscientos treinta pacientes $(72 \%)$ de la población tienen un año o más de permanencia en la clínica de anticoagulación, ellos regis-

Tabla 1. Características generales de la población en terapia de anticoagulación.

\begin{tabular}{|c|c|c|c|c|c|}
\hline Variables & Total & Fibrilación A & ETEV & Embolia Arterial & Prótesis V M \\
\hline Número de pacientes & $319(100 \%)$ & $121(38 \%)$ & $112(35 \%)$ & $30(9.5 \%)$ & $56(17.5 \%)$ \\
\hline Masculino & $108(44 \%)$ & $42(35 \%)$ & $29(26 \%)$ & $17(56.6 \%)$ & $20(35.7 \%)$ \\
\hline Femenino & $211(66 \%)$ & $79(65 \%)$ & $83(74 \%)$ & $13(43.4 \%)$ & $36(64.3 \%)$ \\
\hline Edad & 60.3 & 73.9 & 52.8 & 47.8 & 52.7 \\
\hline Número de visitas & 7 & 7.2 & 6.6 & 6,7 & 7.3 \\
\hline Dosis promedio mg/semana & $29.8 \mathrm{mgW}$ & $24 \mathrm{mgW}$ & $33.3 \mathrm{mgW}$ & $32.2 \mathrm{mgsW}$ & $30.8 \mathrm{mgW}$ \\
\hline $\mathrm{TRT}_{\mathrm{R}}$ & $52 \%$ & $55 \%$ & $49 \%$ & $51 \%$ & $52.6 \%$ \\
\hline $\mathrm{TRT}_{\mathrm{R}}$ menor del 30 & $\begin{array}{l}47(14.7 \%) \\
\mathrm{TRT}_{\mathrm{R}} 19 \%\end{array}$ & $\begin{array}{l}16(13.2 \%) \\
\operatorname{TRT}_{\mathrm{R}} 19 \%\end{array}$ & $\begin{array}{l}16(14.2 \%) \\
\mathrm{TRT}_{\mathrm{R}} 17 \%\end{array}$ & $\begin{array}{l}7(23.3 \%) \\
\operatorname{TRT}_{\mathrm{R}} 21 \%\end{array}$ & $\begin{array}{l}8(14,2 \%) \\
\mathrm{TRT}_{\mathrm{R}} 23 \%\end{array}$ \\
\hline $\mathrm{TRT}_{\mathrm{R}} 30$ y 45 & $\begin{array}{c}70(21.9 \%) \\
\mathrm{TRT}_{\mathrm{R}} 38.8 \%\end{array}$ & $\begin{array}{l}25(20.6 \% \\
\text { TRT }_{\mathrm{R}} 38 \%\end{array}$ & $\begin{array}{l}25(22.3 \%) \\
\mathrm{TRT}_{\mathrm{R}} 37 \%\end{array}$ & $\begin{array}{c}7(23.3 \%) \\
\text { TRT }_{\mathrm{R}} 38.7 \%\end{array}$ & $\begin{array}{l}13(23.2 \%) \\
\mathrm{TRT}_{\mathrm{R}} 39 \%\end{array}$ \\
\hline $\mathrm{TRT}_{\mathrm{R}} 46$ y 70 & $\begin{array}{l}124(38.8 \%) \\
\mathrm{TRT}_{\mathrm{R}} 56 \%\end{array}$ & $\begin{array}{c}48(39.8 \%) \\
\mathrm{TRT}_{\mathrm{R}} 57.5 \%\end{array}$ & $\begin{array}{c}49(43.7 \%) \\
\mathrm{TRT}_{\mathrm{R}} 55.5 \%\end{array}$ & $\begin{array}{l}7(23.3 \%) \\
\mathrm{TRT}_{\mathrm{R}} 57 \%\end{array}$ & $\begin{array}{c}20(35.7 \%) \\
\operatorname{TRT}_{\mathrm{R}} 57.3 \%\end{array}$ \\
\hline $\mathrm{TRT}_{\mathrm{R}}$ mayor del 70 & $\begin{array}{l}78(24,4 \%) \\
\mathrm{TRT}_{\mathrm{R}} 83 \%\end{array}$ & $\begin{array}{c}32(26.4 \%) \\
\text { TRT }_{R} 83 \%\end{array}$ & $\begin{array}{l}22(19.6 \%) \\
\text { TRT }_{R} 83 \%\end{array}$ & $\begin{array}{c}9(30.0 \%) \\
\text { TRT }_{\mathrm{R}} 83.5 \%\end{array}$ & $\begin{array}{l}15(26.7 \%) \\
\mathrm{TRT}_{\mathrm{R}} 80 \%\end{array}$ \\
\hline
\end{tabular}


tran 1704 visitas, 7.4 visitas al año, intervalo promedio de visitas e INR cada 49 días (siete semanas). Ochenta y nueve pacientes (28\%) con ingreso a la clínica de anticoagulación menor a un año, estos pacientes registran 528 visitas y 5.9 visitas en promedio por paciente. El TRT $_{R}$ en pacientes con menos de un año de seguimiento fue de $48 \%$ y para pacientes con más de un año de seguimiento fue $54 \%$.

Con respecto al número de visitas se encontró lo siguiente, 109 (34\%) pacientes presentaron menos de cinco visitas en la clínica de anticoagulación, el $\mathrm{TRT}_{\mathrm{R}}$ fue $50 \%$ y 210 pacientes (66\%) con seis o más visitas con un TRT $_{R}$ de $54 \%$.

Distribución por sexo, 44\% (108) hombres edad promedio 61.5 años, $66 \%$ (211) mujeres, edad 59.7 años. La edad promedio para la población general fue 60.3 años.

El TRT $\mathrm{R}_{\mathrm{R}}$ para el sexo masculino fue $51 \%$ y para el sexo femenino $53 \%$.

Dosis semanal promedio de warfarina $29.8 \mathrm{mg}$. Las dosis extremas menores de $21 \mathrm{mg}$ ( $16 \mathrm{mg} / \mathrm{sem})$ se encontraron en 106 pacientes (33\%) y dosis mayores de $49 \mathrm{mg}(67 \mathrm{mg} / \mathrm{sem})$ en 25 pacientes $(7.8 \%) ; 188$ pacientes $(59.2 \%)$ tenían dosis promedio $(31.4 \mathrm{mg} / \mathrm{sem})$. La dosis semanal de warfarina presentó una relación inversa con la edad, en menores de 45 años la dosis semanal de warfarina fue $37.9 \mathrm{mg}$ y disminuyó a $22.1 \mathrm{mg}$ en mayores de 75 años (Tabla 2). El TRT $\mathrm{R}_{\mathrm{R}}$ en pacientes menores de 55 años fue $48 \%$ y en pacientes mayores de 55 años fue $54 \%$.

Se conformaron cuatro grupos con indicación de terapia con warfarina, así: fibrilación auricular (FA) 38\%(121), enfermedad tromboembólica venosa (ETEV) 35\% (112), prótesis valvulares (PV) $17.5 \%$ (56) y embolia o trombosis arterial (EA) $9.5 \%$ (30) (Figura 1). El TRT $\mathrm{R}_{\mathrm{R}}$ fue diferente en cada grupo: ETEV $49 \%$, para embolia arterial $51 \%$, prótesis valvular $52.6 \%$ y fibrilación auricular $55 \%$. La ETEV comparada con las otras indicaciones tuvo menor $\mathrm{TRT}_{R}$.

Setenta y cuatro pacientes estaban en terapia con warfarina original $\left(\operatorname{coumadin}^{\circledR}\right)(23 \%)$ y $245(77 \%)$ con medicamentos genéricos de warfarina. $\mathrm{El} \mathrm{TRT}_{R}$ con warfarinas genéricas fue $51.7 \%$ y con coumadin ${ }^{\circledR} 53.3 \%$.

Un TRT $_{R}$ de $64 \%$ o más fue alcanzado en 52 pacientes (70.2\%) con coumadin ${ }^{\circledR}, 169$ pacientes $(68.9 \%)$ con warfarinas genéricas y en 221 pacientes (69.2\%) de la muestra total.

De los 2232 registros de INR, INR mayor de 3.5 en 241 $(10.7 \%)$ visitas, INR mayor de 5 en $50(2.24 \%)$ visitas e INR menor de 1.5. en 244 (10.9\%) visitas.

Tabla 2. Dosis semanal y edad / $T R T_{R}$

\begin{tabular}{|l|c|c|c|}
\hline Edad (años) & $\begin{array}{c}\mathbf{N}=\mathbf{3 1 9} \\
(\boldsymbol{\%})\end{array}$ & $\begin{array}{c}\text { Dosis/semanal } \\
(\mathbf{m g s} / \mathbf{W})\end{array}$ & $\begin{array}{c}\mathbf{T R T}_{\mathrm{R}} \\
(\boldsymbol{\%})\end{array}$ \\
\hline Menores de 45 & $65(20.3)$ & 37.9 & 48 \\
$46-55$ & $53(16.6)$ & 33.2 & 48 \\
$56-65$ & $60(18.8)$ & 28.7 & 53 \\
$66-75$ & $51(15.9)$ & 25.7 & 56 \\
Mayores de 75 & $90(28.2)$ & 22.1 & 54 \\
\hline
\end{tabular}

La distribución por valores de INR se encuentra en la Figura 2.

\section{Eventos adversos}

Sangrados menores: 16 pacientes (5\%), de éstos, siete pacientes tenían INR menor de $3.5 \mathrm{TRT}_{\mathrm{R}} 74 \%$, nueve registraron INR mayor de 3.5 con $\mathrm{TRT}_{\mathrm{R}} 43 \%$. Cuatro sangrados menores fueron clínicamente significativos, en todos los pacientes con sangrado menor clínicamente significativo se reportó al menos un INR mayor de 3.5.

Un sangrado menor estuvo asociado con el uso concomitante de antiagregantes plaquetarios dos pacientes $(0.65 \%)$ presentaron sangrado mayor, los cuales se presentaron con INR en rango de sobre anticoagulación.

Se reportó un evento adverso por embolia $(0.32 \%)$ en una paciente con FA sin adherencia al tratamiento e INR menor de 1.5 en el momento del evento.

Los factores asociados a un TRT $\mathrm{R}_{\mathrm{R}}$ bajo aunque no presentan significancia estadística fueron, sexo masculino, enfermedad tromboembólica venosa, uso de warfarina genérica, edad menor de 55 años. Factores asociados con un $\mathrm{TRT}_{\mathrm{R}}$ bajo y con significancia estadística fueron el tiempo menor de un año en la clínica de anticoagulación y menos de 5 visitas en el servicio. Las características de estos factores se presentan en las Tablas 3 y 4.

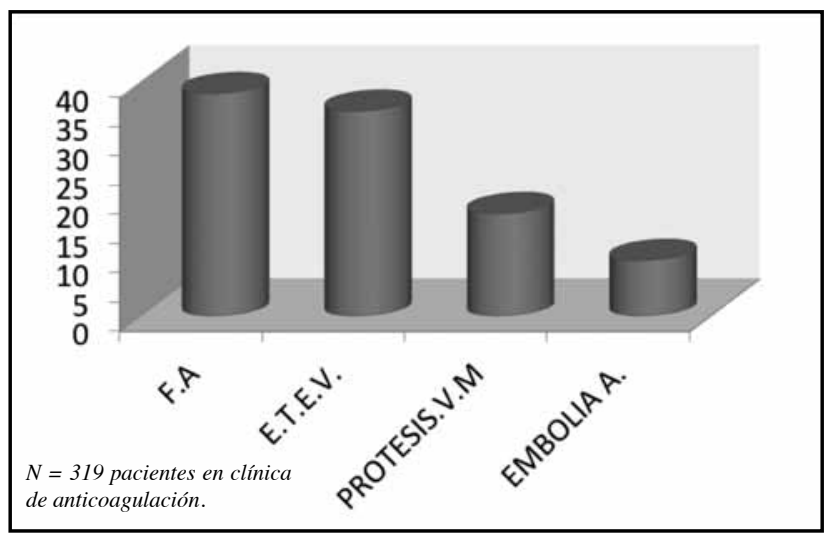

Figura 1. Indicaciones para terapia de anticoagulación con warfarina.

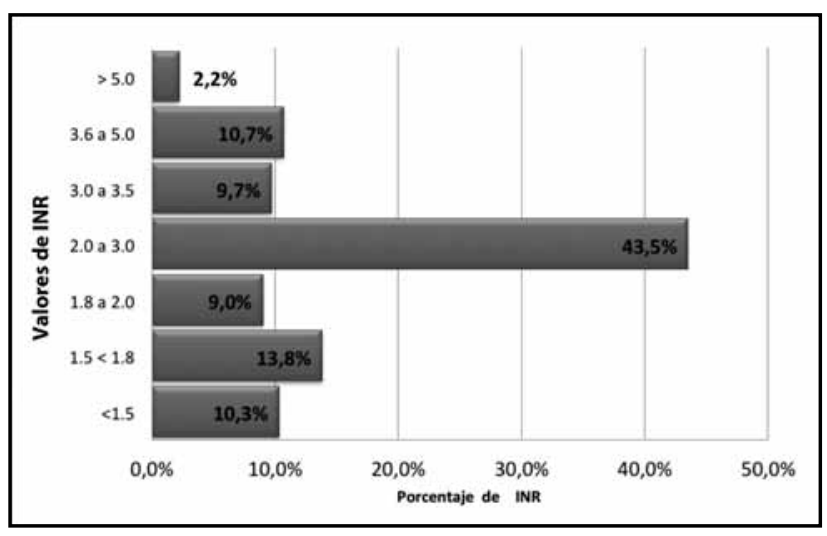

Figura 2. Distribución de INR $n=2-232$ exámenes. 
Tabla 3. Factores asociados a bajo TRT R N=319 pacientes.

\begin{tabular}{|l|c|c|c|}
\hline \multirow{2}{*}{ Sexo } & & TRT (\%) & N \\
& Hombre & 51 & 106 \\
& Mujeres & 53 & 213 \\
\hline \multirow{2}{*}{ Edad } & Menor de 55 a & 48 & 111 \\
& Mayor de 55 a & 54 & 209 \\
\hline Indicación & ETEV & 49 & 112 \\
& Otras & 54 & 207 \\
\hline Medicament & Warfarina & 51.7 & 245 \\
& Coumadin & 53.3 & 74 \\
\hline Visitas & < de 5 visitas & 50 & 109 \\
& $>$ de 5 visitas & 54 & 210 \\
\hline Tiempo en \\
clínica de A & Menor de 1 año & 48 & 89 \\
& Mayor de 1 año & 54 & 230 \\
\hline
\end{tabular}

\section{Discusión}

En la terapia de anticoagulación con warfarina existe una fuerte correlación entre altos valores de $\mathrm{TRT}_{\mathrm{R}} \mathrm{y}$ la reducción en las complicaciones como la trombosis y el sangrado (31). En un metaanálisis de 47 estudios de terapia anticoagulante para pacientes con fibrilación auricular, los estudios aleatorizados mostraron ser superiores a los estudios retrospectivos $\left(\mathrm{TRT}_{\mathrm{R}} 64.9\right.$ vs. 56.4\%); un alto valor de $\mathrm{TRT}_{\mathrm{R}}$ se correlaciona inversamente con presencia de complicaciones (16). En la práctica clínica el TRT $T_{R}$ obtenido usualmente es más bajo al compararlo con el de los estudios clínicos aleatorizados. También se han encontrado diferencias entre el TRT reportado en servicios o clínicas de anticoagulación y el reportado en la práctica comunitaria. Un metaanálisis de ocho estudios con un total de 14 centros participantes y 22237 pacientes tratados con warfarina, en Estados Unidos presentó un $\mathrm{TRT}_{\mathrm{R}}$ promedio de 55\%, los pacientes en clínica de anticoagulación tienen $\mathrm{TRT}_{\mathrm{R}}$ de $63 \%$ comparado con $51 \%$ en pacientes de práctica comunitaria. En Colombia los datos de TRT ${ }_{R}$ registrados son los publicados en el estudio RELY (27), los centros de investigación en Colombia alcanzaron $\mathrm{TRT}_{\mathrm{R}}$ de $53 \%$, nosotros en este estudio para la población con fibrilación auricular reportamos $\mathrm{TRT}_{\mathrm{R}}$ de $55 \%$. Nuestra población consume diferentes marcas de warfarina que puede afectar estos resultados. No se excluyeron los primeros exámenes de INR en la población que inicia terapia anticoagulante y se tomó como rango terapéutico un INR entre dos y tres en todos los pacientes.

Existen diversos reportes de TRT en la literatura, a pesar de las estrategias clínicas el tiempo de rango terapéutico reportado en los diferentes estudios parece no superar el $70 \%$, las clínicas de anticoagulación o servicios de anticoagulación son una estrategia a fin de mejorar el TRT. Estos servicios, clínicas o consultas especializadas, se enfocan en el manejo de la dosis de warfarina para mantener el INR en rango terapéutico; varios estudios han confirmado la validez de esta estrategia, pero no todos han reportado sus resultados en valores de tiempo de rango terapeútico (32). Un estudio en 1993 reportó que 70\% de los pacientes en la clínica de anticoagulación mantenía su INR dentro del rango terapéutico en más de 50\% del tiempo, con una substancial disminución del sangrado y eventos trombóticos (33). Nosotros en este estudio encontramos que $70 \%$ de nuestros pacientes presentan un $\mathrm{TRT}_{\mathrm{R}}$ en promedio de 64\% (40-100).

El TRT $T_{R}$ obtenido en este estudio, sin ser aún un TRT $_{R}$ óptimo y sin alcanzar los publicados en estudios clínicos aleatorizados, nos permite reportar un bajo número de sangrados menores, 16 episodios (5\%), a diferencia del

Tabla 4. Análisis bivariado. Entre los grupos TRT 1 y 2. TRT 1 ( $n=95)$, TRT 2 ( $n=224)$.

\begin{tabular}{|c|c|c|c|c|}
\hline & TRT 1 & TRT2 2 & OR (I.C.) & $\mathbf{P}$ \\
\hline Promedio de edad & $57-97$ & 61.35 & & 0.159 \\
\hline Sexo femenino & $65.3 \%$ & $66.5 \%$ & $0.93(0.56-1.55)$ & 0.79 \\
\hline Embolia arterial & $11 \%$ & $8.5 \%$ & $1.4(0.64-3.1)$ & 0.38 \\
\hline Enf. TEV & $36.8 \%$ & $34.4 \%$ & $1.11(0.67-1.83)$ & 0.67 \\
\hline Fib. auricular (indicación) & 33.7 & $39.7 \%$ & $0.77(0.46-1.28)$ & 0.37 \\
\hline Prótesis valvular & $17.9 \%$ & $17.4 \%$ & $1.03(0.55-1,94)$ & 0.95 \\
\hline Fibrilación auricular & 37.8 & $44.6 \%$ & $0.73(0.45-1.2)$ & 0.27 \\
\hline Warfarina original & $23.2 \%$ & $23.2 \%$ & $1.01(0.56-1.77)$ & 0.89 \\
\hline ASA & $4.2 \%$ & $1.3 \%$ & $3.1(0.7-1.1)$ & 0.24 \\
\hline Clopidogrel & 0 & 0.4 & $2.3(0.1-1.38)$ & 0.5 \\
\hline Nuevos AC & $5.3 \%$ & $1.8 \%$ & $3(0.8-1.1)$ & 0.17 \\
\hline Sangrado mayor & 0 & $0.9 \%$ & $1.16(0.1-13)$ & 0.61 \\
\hline Sangrado menor & $4.2 \%$ & $5.8 \%$ & $0.7(0.23-2.25)$ & 0.76 \\
\hline Visitas 1 & $40 \%$ & $31.3 \%$ & $1.4(0.9-2.4)$ & 0.17 \\
\hline Tiempo en clínica $<1$ año & $36.8 \%$ & $23.7 \%$ & $1.88(1.12-3.1)$ & 0.023 \\
\hline Tiempo clínica > 2 años & 33.7 & $48.6 \%$ & $0.53(0.32-0.88)$ & 0.019 \\
\hline
\end{tabular}


estudio realizado por Builes (34) en Medellín, el cual reportó que $25 \%$ de la población anticoagulada con warfarina presentó sangrado menor, en este estudio no se reportó TRT. Este bajo porcentaje de eventos adversos puede estar en relación con el bajo porcentaje de exámenes de INR mayores a 3.5 e INR mayores a 5.0 (2.2\%) en nuestro estudio. Cannegietes et al. encontraron que el riesgo de sangrado mayor o riesgo de eventos tromboembólicos fue incrementado durante el tiempo en el cual los pacientes estaban por arriba o por abajo del rango del INR, comparado con el tiempo en el cual los pacientes estaban en el INR en rango terapéutico (35). En el estudio de Mattias et al (36) se reportó bajo número de eventos adversos por sangrado en 18361 pacientes seguidos en clínicas de anticoagulación y los valores de INR mayores a 5 fue $0.63 \%$.

La pobre calidad en el manejo de la dosis de warfarina, resulta en una alta proporción de INR por debajo del rango terapéutico; por ejemplo en los primeros tres meses del tratamiento de una trombosis venosa profunda (TVP), que puede traducirse en una alta frecuencia de recurrencia (20, $37)$. Kearon et al evaluaron pacientes con INR en rango de 1.5-2.0 y los compararon con INR de 2-3, los pacientes con INR entre 2 y 3 , presentaron menos recurrencias y no hubo diferencia en el sangrado menor comparado con INR por debajo de rango terapéutico (38). Nosotros reportamos INR por debajo de 1.5 en $10.3 \%$, pero no se encontró recurrencia de eventos trombóticos venosos, y se reportó un evento embólico en paciente con fibrilación auricular.

La edad se correlaciona en forma importante con la variación de la dosis de warfarina como lo mostramos en este estudio, pues de $37.9 \mathrm{mg} / \mathrm{semana}$ para menores de 45 años, disminuyó a $22.1 \mathrm{mg} / \mathrm{semana}$ para mayores de 75 años. Mattias (36) reportó dosis de $43 \mathrm{mg} / \mathrm{semana}$ en pacientes entre 41 y 50 años y $24 \mathrm{mg} / \mathrm{semana}$ en pacientes de 81-90 años. Se requieren bajas dosis de warfarina en pacientes mayores de 65 años. En pacientes mayores de 60 años tratados con warfarina se evidencia una menor depuración de la warfarina por declinación de la función renal con la edad. La edad puede ser el factor más importante predictor del requerimiento de la dosis. Esto podría ser el resultado de menor actividad enzimática en el hígado, uso de medicamentos concomitantes y comorbilidades $(12,14)$. Nuestro estudio no valoró estos aspectos. Nosotros esperamos que estos datos puedan orientar a los profesionales de la salud, para iniciar con dosis bajas de warfarina en los pacientes mayores de 65 años. También encontramos que el $\mathrm{TRT}_{\mathrm{R}}$ es mejor en los pacientes mayores de 55 años, lo que indica que los adultos mayores pueden manejar la warfarina tanto o mejor que los pacientes más jóvenes y la edad por sí sola no debe afectar la decisión de iniciar pacientes en terapia de anticoagulación crónica con warfarina.

Para el cálculo de la dosis, aun usando algoritmos que incluyen variables farmacogenéticas, en $48 \%$ de los pacientes éstos no fueron efectivos para alcanzar un adecuado TRT (39). Los pacientes quienes requieren dosis intermedias de warfarina $(21-49 \mathrm{mg} / \mathrm{s})$ probablemente obtengan poco beneficio de este algoritmo. En nuestro estudio 59.2\% de los pacientes requirieron estas dosis. El algoritmo con componente farmacogénetico tiene mayor utilidad en los pacientes con dosis extremas definidas como dosis de warfarina menor de $21 \mathrm{mg} / \mathrm{s}$ ( $33.9 \%$ de la cohorte estudiada) o mayor de 49 $\mathrm{mg} / \mathrm{s}$ (12.4\% de la cohorte estudiada) (40). Nuestro estudio muestra $33 \%$ de pacientes con dosis menores de $21 \mathrm{mg} / \mathrm{s}$ y $7.8 \%$ dosis mayores de $49 \mathrm{mg}$. La dosis no se asoció a cambios en el TRT R. $_{\text {. }}$

Hay varios factores que están asociados a un bajo $\mathrm{TRT}_{\mathrm{R}}$ en nuestro estudio.

Encontramos que los factores que influyen en tener un bajo $\mathrm{TRT}_{\mathrm{R}}$ fueron, el número bajo de visitas y el tiempo de permanencia en la clínica de anticoagulación menor a un año. Los pacientes con enfermedad tromboembólica venosa presentaron bajos $\mathrm{TRT}_{\mathrm{R}}$ cuando se compararon con los pacientes con otras indicaciones, el sexo masculino, la edad menor de 55 años y el uso de medicamento genérico estuvieron asociados a un bajo $\mathrm{TRT}_{\mathrm{R}}$; sin embargo no alcanzaron significancia estadística, estos factores se deben tener en cuenta cuando se intensifique la monitorización de la terapia de anticoagulación oral con warfarina para optimizar y mejorar el $\mathrm{TRT}_{\mathrm{R}}$.

\section{Limitaciones}

Somos conscientes de las limitaciones de este estudio. El corto tiempo de seguimiento puede afectar la presencia de eventos adversos mayores, un grupo importante de pacientes tenía menos de un año de seguimiento, lo que no hace uniforme a la población. Tratamos de limitar los sesgos al validar un instrumento de recolección de la información, pero el subregistro en las tarjetas de los pacientes puede influir en el reporte de sangrado menor tanto por los pacientes como por los entrevistadores. Los exámenes de INR fueron realizados en diferentes laboratorios, siendo el principal laboratorio el Hospital Universitario San Vicente Fundación, no se especificó la procedencia de todos los INR, lo que pudiera afectar los resultados. Este es un reporte global de pacientes con consumo de varias marcas de warfarina genérica en el mercado y en $23 \%$ warfarina original, lo cual no permite sacar conclusiones de efectividad de un medicamento o fármaco en especial.

\section{Conclusión}

El tiempo de rango terapéutico TRT es una medición útil para establecer la eficacia de la terapia anticoagulante. La meta de $60 \%$ en tiempo de rango terapéutico garantiza menos efectos adversos por sangrado o trombosis. Un número bajo de visitas y anticoagulación menor de un año están asociados a bajo TRT.

\section{Declaración de conflictos de interés}

En la realización de este trabajo no se recibió patrocinio de ninguna índole por parte de la industria farmacéutica, los resultados son tomados y analizados por los investigadores en forma independiente. Los beneficios y patrocinios recibidos por 
los autores en conferencias, participación en eventos científicos no influyeron en la realización de este trabajo.

\section{Referencias}

1. Ageno W, Gallus AS, Wittkowsky A, et al. Oral anticoagulant therapy: Antithrombotic Therapy and Prevention of Thrombosis, 9th ed: American College of Chest Physicians Evidence-Based Clinical Practice Guidelines. Chest. Feb 2012; 141 (2 Suppl): 44S-88S.

2. Fuster V, Ryden LE, Cannom DS, et al. 2011 ACCF/AHA/HRS focused updates incorporated into the ACC/AHA/ESC 2006 Guidelines for the management of patients with atrial fibrillation: a report of the American College of Cardiology Foundation/American Heart Association Task Force on Practice Guidelines developed in partnership with the European Society of Cardiology and in collaboration with the European Heart Rhythm Association and the Heart Rhythm Society. $J$ Am Coll Cardiol. Mar 15 2011; 57 (11): e101-1983.

3. Kearon C,Akl EA, Comerota AJ, et al. Antithrombotic therapy for VTE disease: Antithrombotic Therapy and Prevention of Thrombosis, 9th ed: American College of Chest Physicians Evidence-Based Clinical Practice Guidelines. Chest. Feb 2012; 141 (2 Suppl): e419S-494S.

4. Whitlock RP, Sun JC, Fremes SE, Rubens FD, Teoh KH. American College of Chest P. Antithrombotic and thrombolytic therapy for valvular disease: Antithrombotic Therapy and Prevention of Thrombosis, 9th ed: American College of Chest Physicians Evidence-Based Clinical Practice Guidelines. Chest. Feb 2012; 141 (2 Suppl): e576S-600S.

5. Higashi MK, Veenstra DL, Kondo LM, et al. Association between CYP2C9 genetic variants and anticoagulation-related outcomes during warfarin therapy. JAMA. Apr 3 2002; 287 (13): 1690-1698.

6. Scordo MG, Pengo V, Spina E, Dahl ML, Gusella M, Padrini R. Influence of CYP2C9 and CYP2C19 genetic polymorphisms on warfarin maintenance dose and metabolic clearance. Clin Pharmacol Ther. Dec 2002; 72 (6): 702-710.

7. Loebstein R, Yonath $\mathbf{H}$, Peleg $\mathbf{D}$, et al. Interindividual variability in sensitivity to warfarin--Nature or nurture?. Clin Pharmacol Ther. Aug 2001; 70 (2): 159-164.

8. Holbrook AM, Pereira JA, Labiris R, et al. Systematic overview of warfarin and its drug and food interactions. Arch Intern Med. May 23 2005; 165 (10): 1095-1106.

9. Booth SL, Charnley JM, Sadowski JA, Saltzman E, Bovill EG, Cushman M. Dietary vitamin K1 and stability of oral anticoagulation: proposal of a diet with constant vitamin K1 content. Thromb Haemost. Mar 1997; 77 (3): 504-509.

10. Hylek EM. Paracetamol (acetaminophen) and warfarin interaction: unraveling the pivotal role of the vitamin K cycle. Thromb Haemost. Vol 92. Germany 2004: 672-673.

11. Self TH, Reaves AB, Oliphant CS, Sands C. Does heart failure exacerbation increase response to warfarin? A critical review of the literature. Curr Med Res Opin. Nov 2006; 22 (11): 2089-2094.

12. Mungall D, White R. Aging and warfarin therapy. Ann Intern Med. Nov 15 1992; 117 (10): 878-879.

13. Dreisbach AW, Japa S, Gebrekal AB, et al. Cytochrome P4502C9 activity in end-stage renal disease. Clin Pharmacol Ther. Vol 73. United States 2003: 475477.

14. Garcia D, Regan S, Crowther M, Hughes RA, Hylek EM. Warfarin maintenance dosing patterns in clinical practice: implications for safer anticoagulation in the elderly population. Chest. Jun 2005; 127 (6): 2049-2056.

15. Stafford DW. The vitamin K cycle. J Thromb Haemost. Aug 2005; 3 (8): 1873 1878 .

16. Wan Y, Heneghan C, Perera R, et al. Anticoagulation control and prediction of adverse events in patients with atrial fibrillation: a systematic review. Circ Cardiovasc Qual Outcomes. Nov 2008; 1 (2) : 84-91.

17. Baker WL, Cios DA, Sander SD, Coleman CI. Meta-analysis to assess the quality of warfarin control in atrial fibrillation patients in the United States. $J$ Manag Care Pharm. Apr 2009; 15 (3): 244-252.

18. Kirkwood TB. Calibration of reference thromboplastins and standardisation of the prothrombin time ratio. Thromb Haemost. Jun 28 1983; 49 (3): 238-244.

19. Poller L. International Normalized Ratios (INR): the first 20 years. J Thromb Haemost. Jun 2004; 2 (6): 849-860.

20.Palareti G, Legnani C, Guazzaloca G, et al. Risks factors for highly unstable response to oral anticoagulation: a case-control study. Br J Haematol. Apr 2005; 129 (1): $72-78$.

21. White HD, Gruber M, Feyzi J, et al. Comparison of outcomes among patients randomized to warfarin therapy according to anticoagulant control: results from SPORTIF III and V. Arch Intern Med. Feb 12 2007; 167 (3): 239-245.

22. Connolly SJ, Laupacis A, Gent M, Roberts RS, Cairns JA. Joyner C. Canadian Atrial Fibrillation Anticoagulation (CAFA) Study. J Am Coll Cardiol. Aug 1991; 18 (2): 349-355

23. Rosendaal FR, Cannegieter SC, van der Meer FJ, Briet E. A method to determine the optimal intensity of oral anticoagulant therapy. Thromb Haemost. Mar 1 1993; 69 (3): 236-239.

24. Connolly SJ, Ezekowitz MD, Yusuf S, et al. Dabigatran versus warfarin in patients with atrial fibrillation. N Engl J Med. Sep 17 2009; 361 (12): 1139-1151.

25. Granger CB, Alexander JH, McMurray JJ, et al. Apixaban versus warfarin in patients with atrial fibrillation. N Engl J Med. Sep 15 2011; 365 (11): 981-992.

26. Investigators E-P, Buller HR, Prins MH, et al. Oral rivaroxaban for the treatment of symptomatic pulmonary embolism. N Engl J Med. Apr 5 2012; 366 (14): 1287-1297.

27. Wallentin L, Yusuf S, Ezekowitz MD, et al. Efficacy and safety of dabigatran compared with warfarin at different levels of international normalised ratio control for stroke prevention in atrial fibrillation: an analysis of the RE-LY trial. Lancet. Sep 18 2010; 376 (9745): 975-983.

28. Schulman S, Kearon C. Subcommittee on Control of Anticoagulation of the Scientific and Standardization Committee of the International Society on Thrombosis and $\mathrm{H}$. Definition of major bleeding in clinical investigations of antihemostatic medicinal products in non-surgical patients. J Thromb Haemost. Apr 2005; 3 (4): 692-694.

29. Buller HR, Davidson BL, Decousus H, et al. Subcutaneous fondaparinux versus intravenous unfractionated heparin in the initial treatment of pulmonary embolism. N Engl J Med. Oct 30 2003; 349 (18): 1695-1702.

30. Decousus H, Tapson VF, Bergmann JF, et al. Factors at admission associated with bleeding risk in medical patients: findings from the IMPROVE investigators. Chest. Jan 2011; 139 (1): 69-79.

31. Connolly SJ, Pogue J, Eikelboom J, et al. Benefit of oral anticoagulant over antiplatelet therapy in atrial fibrillation depends on the quality of international normalized ratio control achieved by centers and countries as measured by time in therapeutic range. Circulation. Nov 11 2008; 118 (20): 2029-2037.

32. Lader E, Martin N, Cohen G, et al. Warfarin therapeutic monitoring: is $70 \%$ time in the therapeutic range the best we can do?. J Clin Pharm Ther. Dec 162011.

33. Cortelazzo S, Finazzi G, Viero P, et al. Thrombotic and hemorrhagic complications in patients with mechanical heart valve prosthesis attending an anticoagulation clinic. Thromb Haemost. Apr 1 1993; 69 (4): 316-320.

34. Builes CE, Arango A, Cano WM. Frecuencia de sangrado en pacientes con enfermedades cardiovasculares anticoagulados con warfarina genérica vs. Coumadin. Acta Medica Colombiana. 2010;35:175-178

35 Cannegieter SC, Rosendaal FR, Briet E. Thromboembolic and bleeding complications in patients with mechanical heart valve prostheses. Circulation. Feb 1994; 89 (2): 635-641.

36. Wieloch M, Sjalander A, Frykman V, Rosenqvist M, Eriksson N, Svensson PJ.Anticoagulation control in Sweden: reports of time in therapeutic range, major bleeding, and thrombo-embolic complications from the national quality registry AuriculA. Eur Heart J. Sep 2011; 32 (18): 2282-2289.

37.Palareti G, Legnani C, Cosmi B, Guazzaloca G, Cini M, Mattarozzi S. Poo anticoagulation quality in the first 3 months after unprovoked venous thromboembolism is a risk factor for long-term recurrence. J Thromb Haemost. May 2005; 3 (5): 955-961.

38. Kearon C, Ginsberg JS, Kovacs MJ, et al. Comparison of low-intensity warfarin therapy with conventional-intensity warfarin therapy for long-term prevention of recurrent venous thromboembolism. N Engl J Med. Aug 14 2003; 349 (7): 631 639.

39. Finkelman BS, Gage BF, Johnson JA, Brensinger CM, Kimmel SE. Genetic warfarin dosing: tables versus algorithms. J Am Coll Cardiol. Feb 1 2011; 57 (5): 612-618.

40. International Warfarin Pharmacogenetics C, Klein TE, Altman RB, et al. Estimation of the warfarin dose with clinical and pharmacogenetic data. $N$ Engl J Med. Feb 19 2009; 360 (8): 753-764. 